\title{
Reduction of Perioperative Blood Loss and
}

Operating Time for Arthroscopic Rotator Cuff Repair by Intravenous Administration of Tranexamic Acid

Shinji Kawaguchi

National Hospital Organization Kochi National Hospital

Shoji Fukuta ( $\sim$ shojifc001@yahoo.co.jp )

Kochi National Hospital https://orcid.org/0000-0003-2658-1811

\section{Masashi Kano}

National Hospital Organization Kochi National Hospital

Koichi Sairyo

Department of Orthopedics, Tokushima University

Research article

Keywords: Reduction of perioperative blood loss, arthroscopic rotator cuff repair, intravenous administration of tranexamic acid

Posted Date: November 1st, 2021

DOI: https://doi.org/10.21203/rs.3.rs-1010175/v1

License: (9) (1) This work is licensed under a Creative Commons Attribution 4.0 International License. Read Full License 


\section{Abstract}

(Background) Tranexamic acid (TXA) is widely used in hip and knee arthroplasty to reduce perioperative bleeding. Recently, its use has been expanded to arthroscopic surgery. The purpose of this study was to evaluate the efficacy of preoperative use of TXA in arthroscopic rotator cuff repair (RCR).

(Methods) A cohort comprising 129 consecutive patients who underwent arthroscopic primary RCR at our institution was retrospectively investigated according to whether they received TXA (April 2018 to December 2020, TXA group, $n=64$ ) or did not receive TXA (April 2016 to March 2018, non-TXA group, $\mathrm{n}=65$ ). TXA was administered at a dose of $1 \mathrm{~g}$ intravenously. Rotator cuff tears were repaired by the suture bridge technique. Videos of the arthroscopic procedures were reviewed and rated for visual clarity using a 10-point numeric rating scale. Arthroscopic procedures were divided into glenohumeral, resection of bursal tissue and acromioplasty, and RCR steps. Each step was rated separately. Age, sex, body mass index, hemoglobin level before and on days 1 and 7 after surgery, operating time, mean arterial pressure, tear size, and number of anchors used for cuff repair were compared between the two groups.

(Results) There were no statistically significant differences in the patient demographic data. The operating time was significantly shorter in the TXA group than in non-TXA group $(97.8 \pm 21.8$ min vs $116.2 \pm 26.0 \mathrm{~min})$. The clarity of the visual field was similar between the two groups during the glenohumeral phase but was significantly higher in the TXA group during the resection of bursal tissue and acromioplasty and RCR phases. Hemoglobin level was not significantly different between the groups on postoperative day 1 but was significantly higher in the TXA group on day 7 .

(Conclusion) Administration of a single intravenous dose of TXA improved visual clarity in arthroscopic RCR, decreased the total operating time, and reduced hemoglobin loss on postoperative day 7 .

\section{Background}

Arthroscopic repair has been one of the major surgical options for rotator cuff tears for the past 2 decades. Arthroscopic technique has evolved from single-row repair to double-row repair with the suture bridge technique in an attempt to prevent further tears after arthroscopic rotator cuff repair (RCR) 1,2). Recent procedures have become more complicated with the increase in the numbers of anchors used.

Unlike knee arthroscopy, a pneumatic tourniquet cannot be applied in shoulder arthroscopy. Therefore, control of intraoperative bleeding is essential for a safe and secure procedure. Pressure-controlled irrigation pumps 3,4), thermal electrocautery devices 5), hypotensive anesthesia 6), and addition of epinephrine to the irrigation fluid 7-9) are widely used to control intraoperative bleeding.

Tranexamic acid (TXA) is a synthetic antifibrinolytic agent that is often used to reduce perioperative bleeding and the need for transfusion in patients undergoing hip and knee arthroplasty 10-11). Recently, its use has been expanded to knee and shoulder arthroscopic surgery, including anterior cruciate ligament reconstruction 12,13), partial meniscectomy 14), and RCR 15). We hypothesized that intravenous 
administration of TXA would reduce intraoperative bleeding during arthroscopic RCR, improve visual clarity, and reduce operating time. This study evaluated the safety and efficacy of preoperative use of TXA in arthroscopic RCR.

\section{Methods}

A cohort of 129 patients who underwent arthroscopic primary RCR by the same surgeon (S.F.) between April 2016 and December 2020 were retrospectively investigated. Since April 2018, we have used intravenous TXA to reduce intraoperative bleeding during arthroscopic procedures. After induction of anesthesia, $1 \mathrm{~g}$ of TXA was administered in all patients except in those with a history of deep venous thrombosis, pulmonary embolism, stroke, or cardiac stent placement and in those with allergy to TXA.

The inclusion criteria were as follows: full-thickness supraspinatus and/or infraspinatus tear confirmed by arthroscopy; partial-thickness tear converted to full-thickness tear before repair; and arthroscopic repair by the suture bridge technique. The exclusion criteria included partial repair, single-row or conventional double-row repair, tear involving the subscapularis tendon, a concomitant glenohumeral procedure such as labral repair or capsular release, and revision surgery. Patients treated with anticoagulant agents were also excluded. Patients who underwent concomitant tenotomy or tenodesis of the long head of biceps were not excluded. A total of 137 patients met the eligibility criteria and were divided into 2 groups according to whether or not they received TXA: 64 consecutive patients received TXA from April 2018 to December 2020 (the TXA group), and 65 consecutive patients did not receive TXA from April 2016 to March 2018 (the non-TXA group).

All procedures were performed with the patient in the beach-chair position under general anesthesia with the upper extremity fixed by a limb positioner. Interscalene regional nerve block was not performed in all cases. The irrigation fluid pressure was kept at $40 \mathrm{mmHg}$ by a pressure-controlled pump. Epinephrine was not added to the irrigation bags. Blood pressure was measured in the contralateral extremity using an automatic blood pressure cuff at 5-min intervals intraoperatively. To avoid cerebral ischemia, hypotensive anesthesia was not applied. Blood pressure was controlled within the normal range during surgery.

Diagnostic arthroscopy was performed to evaluate the rotator cuff and associated pathology. Intraarticular lesions were treated appropriately. The arthroscope was then placed into the subacromial space and a complete bursectomy and acromioplasty were performed in all patients. Acromioplasty was limited to removal of a large acromial spur. Minimal release of the coracoacromial ligament was performed if the undersurface of the acromion was free of osteophytes. The torn edge of the rotator cuff was debrided and the footprint was refreshed with a shaver to allow bleeding from cancellous bone. A 5.5-mm anchor preloaded with 3 No. 2 FiberWire sutures (5.5-mm PEEK Corkscrew FT; Arthex, Naples, FL) was inserted at the articular margin of the greater tuberosity. One or two anchors were used for the medial row depending on the size of the tear. A No. 2 FiberLink (Arthex) was passed through the tendon lateral to the musculotendinous junction using a suture passing device (ExpresSew II; Depuy Mitek, Raynham, MA) and one limb of the three sutures from each medial anchor was relayed at the same time with a FiberLink. 
After each pass, pairs of suture limbs from each medial anchor were retrieved and suture bridges were created over the tendon with 2 lateral row anchors (5.5-mm SwiveLock; Arthex) inserted 10-15 mm distal to the greater tuberosity. After the suture bridges were completed, the remaining medial sutures were tied in a horizontal mattress fashion to press down and secure the cuff to the footprint. If a dog-ear deformity was present after completing the repair, it was treated using a preloaded additional eyelet suture on the lateral anchor

Information on age, sex, height, weight, body mass index, and preoperative and postoperative hemoglobin levels was collected from the clinical records, whereas data on operating time, mean arterial pressure, tear size, and the number of anchors were obtained from the operation notes. Postoperative hemoglobin levels were obtained on days 1 and 7 after surgery. Loss of hemoglobin was calculated by subtracting the levels on postoperative days 1 and 7 from the preoperative value.

Videos of the arthroscopic procedures were reviewed by a single shoulder fellow who was blinded to whether or not TXA was used. Visibility during the arthroscopic procedures was scored using a 10-point numeric rating scale (NRS; 10, best; Figure 1). The arthroscopic procedures were divided into glenohumeral (GH) arthroscopy, arthroscopic subacromial decompression (ASD) including resection of bursal tissue and acromioplasty, and RCR steps. Each step was rated separately.

The statistical analysis included patient demographics, operating time, mean blood pressure, tear size, number of anchors used, postoperative hemoglobin loss, and the NRS score for visual clarity during each arthroscopic step. Descriptive statistics are presented as the mean \pm standard deviation for continuous data and as the median when nonparametric testing was necessary. The two-sample unpaired Student's $t$-test was used to compare continuous variables. The NRS score was compared between the study groups using the non-parametric Mann-Whitney test. Categorical variables were compared using the chisquared test. All analyses were performed using IBM SPSS version 21 (IBM Corp., Armonk, NY). A P-value $<0.05$ was considered statistically significant.

\section{Results}

There were no statistically significant differences in the demographic data, including age, sex, body mass index, and preoperative hemoglobin level, between the two groups (Table 1). Operating time was significantly shorter in the TXA group than in the non-TXA group (97.8 $\pm 21.8 \mathrm{~min}$ vs $116.2 \pm 26.0 \mathrm{~min}$ ). There was no significant between-group difference in mean arterial blood pressure. There was also no significant difference in tear size, number of anchors used, or the frequency of concomitant biceps tenodesis or tenotomy. The comparison of intraoperative variables between the 2 groups is summarized in Table 2. 
Table 1

Patient demographics

\begin{tabular}{|llll|}
\hline & Non-TXA group & TXA group & P-value \\
\hline Patients, $\mathrm{n}$ & 65 & 64 & \\
\hline Sex (male: female) & $38: 27$ & $41: 23$ & 0.514 \\
\hline Age (years) & $64.0 \pm 8.7$ & $64.9 \pm 8.7$ & 0.570 \\
\hline Height (cm) & $160.6 \pm 9.4$ & $162.0 \pm 8.9$ & 0.378 \\
\hline Weight (kg) & $62.4 \pm 10.0$ & $63.7 \pm 12.4$ & 0.490 \\
\hline Body mass index & $24.2 \pm 3.4$ & $24.2 \pm 3.8$ & 0.991 \\
\hline TXA, tranexamic acid & & \\
\hline
\end{tabular}

Table 2

Operative variables in the two study groups

\begin{tabular}{|llll|}
\hline & Non-TXA group & TXA group & P-value \\
\hline Operating time $(\mathrm{min})$ & $116.2 \pm 26.0$ & $97.8 \pm 21.8$ & 0.000 \\
\hline Systolic blood pressure $(\mathrm{mmHg})$ & $105.9 \pm 8.7$ & $104.3 \pm 9.4$ & 0.324 \\
\hline Diastolic blood pressure $(\mathrm{mmHg})$ & $63.5 \pm 7.2$ & $62.1 \pm 7.2$ & 0.275 \\
\hline Mean arterial pressure $(\mathrm{mmHg})$ & $84.7 \pm 6.9$ & $83.2 \pm 7.5$ & 0.246 \\
\hline Tear size $(\mathrm{mm})$ & $15.0 \pm 6.7$ & $16.4 \pm 7.1$ & 0.264 \\
\hline Number of anchors used & $3.5 \pm 0.6$ & $3.6 \pm 0.6$ & 0.308 \\
\hline Concomitant procedures (none, tenodesis, tenotomy) & $45,18,2$ & $49,15,0$ & 0.296 \\
\hline TXA, tranexamic acid & & & \\
\hline
\end{tabular}

The NRS score for clarity of the visual field was similar in the two groups during the GH step but was significantly higher in the TXA group than in the non-TXA group during ASD and RCR (Table 3). NRS scores were lowest during ASD in both groups. There was no significant between-group difference in hemoglobin loss on postoperative day 1 ; however, the decrease in the hemoglobin level was significantly greater in the TXA group on postoperative day 7 (Table 4). 
Table 3

Visual clarity during arthroscopic rotator cuff repair

\begin{tabular}{|llll|}
\hline & Non-TXA group & TXA group & P-value \\
\hline Glenohumeral procedure, median (row score) & $8(4-10)$ & $8(4-9)$ & 0.268 \\
\hline Subacromial decompression, median, (row score) & $6(3-9)$ & $7(4-9)$ & 0.000 \\
\hline Rotator cuff repair, median (row score) & $7(3-9)$ & $8(5-10)$ & 0.001 \\
\hline TXA, tranexamic acid & & & \\
\hline
\end{tabular}

Table 4

Perioperative hemoglobin levels and postoperative hemoglobin loss

\begin{tabular}{|lccc|}
\hline & Non-TXA group & TXA group & P-value \\
\hline Preoperative hemoglobin level $(\mathrm{g} / \mathrm{dL})$ & $14.3 \pm 1.3$ & $14.5 \pm 1.5$ & 0.427 \\
\hline Hemoglobin level on POD $1(\mathrm{~g} / \mathrm{dL})$ & $13.0 \pm 1.4$ & $13.4 \pm 1.5$ & 0.122 \\
\hline Hemoglobin level on POD 7 (g/dL) & $12.8 \pm 1.2$ & $13.4 \pm 1.3$ & 0.005 \\
\hline Hemoglobin loss on POD 1 (g/dL) & $1.3 \pm 0.8$ & $1.1 \pm 0.9$ & 0.172 \\
\hline Hemoglobin loss on POD 7 $(\mathrm{g} / \mathrm{dL})$ & $1.5 \pm 0.8$ & $1.1 \pm 0.8$ & 0.004 \\
\hline POD, postoperative day; TXA, tranexamic acid & & \\
\hline
\end{tabular}

\section{Discussion}

Control of intraoperative bleeding is critical in arthroscopic shoulder surgery because uncontrolled bleeding may lead to an incorrect procedure being performed and an extended operating time. In the past, epinephrine has been added to the irrigation fluid to improve visual clarity 7-9). In a prospective, randomized, double-blind controlled study by Jensen et al. that involved 54 shoulder arthroscopies, addition of epinephrine to the irrigation fluid reduced intra-articular bleeding and improved visualization without any adverse events 7). Another prospective randomized study by Avery et al. 8) compared surgeon-rated visualization using irrigation fluid with and without epinephrine in 83 arthroscopic shoulder procedures and found that addition of epinephrine significantly improved visualization. Like the study reported by Jansen et al 7), their study included various types of arthroscopic surgery, including RCR, and did not demonstrate a difference in operating time. Montfoort et al. 9) performed a similar double-blind study to determine the effect of epinephrine-saline irrigation on the clarity of view during shoulder arthroscopy procedures. In contrast with the previous studies, they reported a significant reduction in total operating time of $15 \mathrm{~min}$ in addition to improved visual clarity. 
Previous studies have demonstrated that TXA is effective in reducing total blood loss, the transfusion rate, and the volume of blood drained in patients undergoing total knee and total hip arthroplasty without increasing the complication rate 16-18). Although TXA has not been applied in arthroscopic surgery, which rarely requires blood transfusion, its administration in arthroscopic anterior cruciate ligament (ACL) reconstruction has been a focus of interest in the past few years. A randomized controlled trial that included $105 \mathrm{ACL}$ reconstructions found that administration of TXA reduced the amount of postoperative hemarthrosis and decreased the need for aspiration of the knee 12). Another prospective study demonstrated that a single intravenous dose of TXA in patients undergoing ACL reconstruction improved both range of motion at the knee and quadriceps strength, reduced hemarthrosis, and decreased the volume of blood removed by[suction drainage 13].

Given that intraoperative bleeding is not a major issue in knee arthroscopic surgery, evaluation of the effect of TXA has focused mainly on postoperative bleeding and related complications, including soft tissue swelling and pain. However, unlike with knee arthroscopy, whether or not administration of TXA improves visual clarity by decreasing intraoperative bleeding is a major topic of research. For example, Liu et al. 15) performed a double-blind randomized trial in which they investigated 72 patients who underwent arthroscopic suture bridge repair with intravenous administration of TXA and reported improved visual clarity with reduced levels of subjective pain and analgesic consumption. They rated visual clarity using a 3-point NRS every 15 min but showed only the average rating score, whereas in the present study, the arthroscopic procedure was divided into 3 steps and each step was evaluated separately. There was no significant between-group difference in the NRS score for visual clarity during the glenohumeral step. However, the score for visual clarity was significantly higher during the subacromial step with the maximal difference between the study groups noted during the ASD step, which was lowest in both groups. It is difficult to coagulate bleeding from bone marrow during acromioplasty or anchor insertion when using a thermal electrocautery device; therefore, TXA may be very effective in controlling bleeding from this site. Furthermore, poor visual clarity due to bleeding from an anchor hole often interferes with smooth management of the anchor sutures, which leads to a longer operating time. In this study, the operating time was shortened by administration of TXA, whereas Liu et al. 15) reported that the operating time was similar between their study groups. This discrepancy might be explained by the fact that ASD was performed in all patients in the present study but in only 5 of 72 patients in their study. According to our findings, ASD was the step in arthroscopic RCR that was most vulnerable to bleeding. Therefore, whether or not ASD is performed might explain these conflicting results regarding the ability of TXA to shorten the operating time. Other potential explanations include differences in patient positioning, whether or not an interscalene nerve block was used, and the initial irrigation pressure setting.

The hemoglobin level decreased to a similar extent on postoperative day 1 in both of our study groups. Although Liu et al. did not report on hemoglobin levels, they found no significant difference in total blood loss as estimated from the decrease in hemoglobin, which is consistent with our findings. The ability of TXA to prevent bleeding was sufficient to improve visual clarity but might not be enough to reduce loss of hemoglobin in arthroscopic RCR. Interestingly, we found a significant difference in the amount of 
hemoglobin loss by postoperative day 7 according to whether or not TXA was administered. Therefore, administration of a single intravenous dose of TXA reduced both postoperative and intraoperative bleeding.

This study has several limitations. First, it had a retrospective non-randomized design, which might have introduced a degree of bias in terms of patient selection and surgical procedures. However, we did not change our indications for arthroscopic RCR or the surgical techniques used during the study period. Furthermore, the surgeon had more than 15 years of experience in shoulder arthroscopy and was proficient in arthroscopic RCR. Therefore, any bias in terms of surgical skill was negligible. Moreover, visual clarity was scored by an observer who was blinded to all information regarding administration of TXA, which minimized any rating bias. Second, visibility was evaluated only once by a single rater, and the reliability and reproducibility of NRS rating was not determined. However, evaluation of visual clarity using videos of entire arthroscopic procedures would have been too time-consuming, and repeated rating by several observers would be close to impossible. Finally, we did not evaluate subjective pain in the immediate postoperative period on account of a change in our postoperative pain relief protocol during the study period.

\section{Conclusion}

Administration of a single intravenous dose of TXA improved visual clarity when performing arthroscopic RCR and decreased the total operating time with less loss of hemoglobin by postoperative day 7 . This method is simple and safe and may be an alternative to adding epinephrine to the irrigation fluid in order to improve visual clarity in arthroscopic shoulder surgery.

\section{Abbreviations}

TXA: tranexamic acid

RCR: rotator cuff repair

$\mathrm{GH}$ : glenohumeral

ASD: arthroscopyic subacromial decompression

NRS: numeric rating scale

\section{Declarations}

\section{Acknowledgement}

Not applicable 


\section{Ethic approval and consent to participate}

This study was approved by the Institutional Review Board of National Hospital Organization Kochi National Hospital (R2-15). The requirement for informed consent was waved for this study owing to its retrospective nature. Patients were given the opportunity to opt out of this study

\section{Consent for publication}

Not applicable

\section{Availability of data and materials}

The data and materials used and analyzed during the current study are available from the corresponding author on reasonable request.

\section{Competing interests}

The Authors declare that there is no competing interest.

\section{Funding}

Not applicable

\section{Authors' contributions}

SK analyzed the data and drafted the manuscript. SF performed all arthroscopic surgeries and was involved in writing and editing of the article. MK collected the data. KS contributed to conception and study design of the article. All authors read and approved the final manuscript.

\section{References}

1. Chen M, Xu W, Dong Q, Huang Q, Xie Z, Mao Y. Outcomes of single-row versus double-row arthroscopic rotator cuff repair: a systematic review and meta-analysis of current evidence. Arthroscopy 2013; 29: 1437-1449.

2. Hein J, Reilly JM, Chae J, Maerz T, Anderson K. Retear Rates After Arthroscopic Single-Row, DoubleRow, and Suture Bridge Rotator Cuff Repair at a Minimum of 1 Year of Imaging Follow-up: A Systematic Review. Arthroscopy 2015; 31: 2274-2281. 
3. Ampat G, Bruguera J, Copeland SA. Aquaflo pump vs FMS 4 pump for shoulder arthroscopic surgery. Ann R Coll Surg Engl 1997; 79: 341-344.

4. Ogilvie-Harris DJ, Weisleder L. Fluid pump systems for arthroscopy: A comparison of pressure control versus pressure and flow control. Arthroscopy 1995; 11: 591-595.

5. Nho SJ, Freedman KB, Bansal SL, et al. The effect of radiofrequency energy on non weight-bearing areas of bone following shoulder and knee arthroscopy. Orthopedics 2005; 28: 392-399.

6. Morrison DS, Schaefer RK, Friedman RL. The relationship between subacromial space pressure, blood pressure, and visual clarity during arthroscopic subacromial decompression. Arthroscopy 1995; 11: 557-560.

7. Jensen KH, Werther K, Stryger V, Schultz K, Falkenberg B. Arthroscopic shoulder surgery with epinephrine saline irrigation. Arthroscopy 2001; 17: 578-581.

8. Avery DM, Gibson BW, Carolan GF. Surgeon-rated visualization in shoulder arthroscopy: a randomized blinded controlled trial comparing irrigation fluid with and without epinephrine. Arthroscopy 2015; 31: 12-18.

9. Montfoort DO, van Kampen PM, Huijsmans PE. Epinephrine diluted saline-irrigation fluid in arthroscopic shoulder surgery: A significant improvement of clarity of visual field and shortening of total operation time. A randomized controlled trial. Arthroscopy 2016; 32: 436-444.

10. Zufferey P, Merquiol F, Laporte S, Decousus H, Mismetti P, Auboyer C, et al. Do antifibrinolytics reduce allogeneic blood transfusion in orthopedic surgery. Anes-thesiology 2006; 105: 1034-1046.

11. Kagoma YK, Crowther MA, Douketis J, Bhandari M, Eikelboom J, Lim W. Use of antifibrinolytic therapy to reduce transfusion in patients undergoing orthopedic surgery: A systematic review of randomized trials. Thromb Res 2009; 123:687-696.

12. Karaaslan F, Karaoglu S, Yurdakul E. Reducing intra-articular hemarthrosis after arthroscopic anterior cru-ciate ligament reconstruction by the administration of intravenous tranexamic acid: A prospective, random-ized controlled trial. Am J Sports Med 2015; 43: 2720-2726.

13. Felli $L$, Revello $S$, Burastero $G$, et al. Single intravenous administration of tranexamic acid in anterior cruciate ligament reconstruction to reduce postoperative hemarthrosis and increase functional outcomes in the early phase of postoperative rehabilitation: A randomized controlled trial. Arthroscopy 2019; 35: 149-157.

14. Nugent M, May JH, Parker JD, et al. Does tranexamic acid reduce knee swelling and improve early function following arthroscopic meniscectomy? A double-blind randomized controlled trial. Orthop J Sports Med 2019; 7:2325967119866122.

15. Liu YF, Hong CK, Hsu KL, et al. Intravenous administration of tranexamic acid significantly improved clarity of the visual field in arthroscopic shoulder surgery. A prospective, double-blind, and randomized controlled trial. Arthroscopy 2020; 36: 640-647.

16. Jansen AJ, Andreica S, Claeys M, D'Haese J, Camu F, Jochmans K. Use of tranexamic acid for an effective blood conservation strategy after total knee arthroplasty. Br J Anaesth 1999; 83: 596-601. 
17. Yang ZG, Chen WP, Wu LD. Effectiveness and safety of tranexamic acid in reducing blood loss in total knee arthroplasty: a meta-analysis. J Bone Joint Surg Am. 2012; 94: 1153-9.

18. Noordin S, Waters TS, Garbuz DS, Duncan CP, Masri BA. Tranexamic acid reduces allogenic transfusion in revision hip arthroplasty. Clin Orthop Relat Res 2011; 469: 541-546.

\section{Figures}

(A)

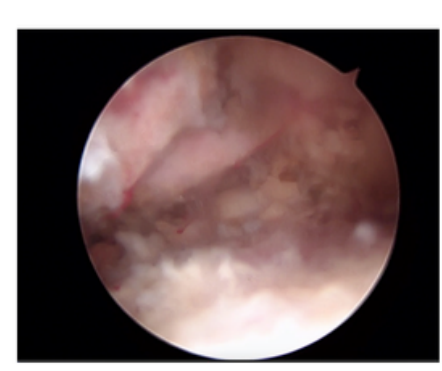

(B)

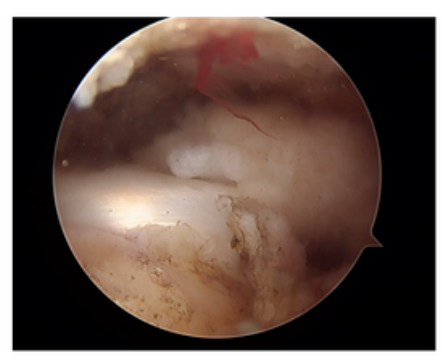

(C)

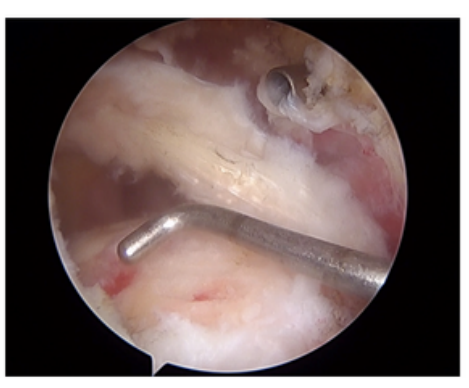

(D)

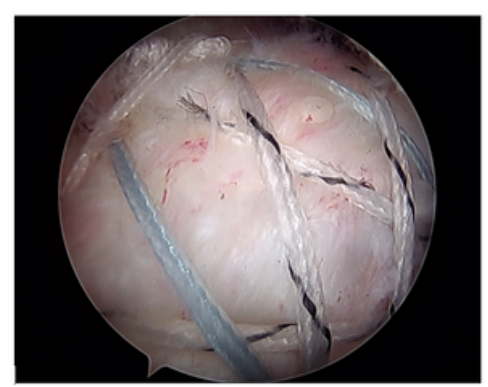

\section{Figure 1}

Representative arthroscopic images viewed through the posterolateral viewing portal in the subacromial space. The view was scored using the numeric rating scale (NRS) method. NRS scores are shown for values of (A) 4, (B) $5,(C) 7$, and (D) 9. 